DOI: $10.24850 /$ j-tyca-2018-05-08

Artículos

\title{
Eliminación de pesticidas mediante un biorreactor de membrana y dos tiempos diferentes de retención celular
}

\author{
Edgardo Vásquez ${ }^{1}$ \\ Arturo Trapote ${ }^{2}$ \\ Daniel Prats ${ }^{3}$
}

${ }^{1}$ Universidad de Alicante, San Vicente del Raspeig, Alicante, España / Grupo de Investigación Ambiental, Tecnológica y Energética de Coclé (Giatec), Universidad Tecnológica de Panamá, Ciudad de Panamá, Panamá, edgardovasquez1990@gmail.com, evr13@alu.ua.es https://orcid.org/00000001-5251-9662

2 Universidad de Alicante, San Vicente del Raspeig, Alicante, España, atj@ua.es https://orcid.org/0000-0003-2084-270X

3 Universidad de Alicante, San Vicente del Raspeig, Alicante, España, prats@ua.es https://orcid.org/0000-0002-5727-7479

Autor para correspondencia: Edgardo Vásquez, edgardovasquez1990@gmail.com, evr13@alu.ua.es

\section{Resumen}

En esta investigación se evaluó la eficiencia de un biorreactor de membrana (MBR, por sus siglas en inglés), para eliminar pesticidas. Además, se comprobó que la presencia de pesticidas no influyera en la eficiencia de eliminación de materia orgánica. Se estudió un total de 16 pesticidas, con los que se dopó agua residual urbana sintética. La investigación se llevó a cabo en una planta MBR a escala piloto, operada a tiempos de retención celular (TRC) de 30 y 60 días, carga másica de $0.23 \mathrm{kgDQO} \cdot \mathrm{kgSSV}^{-1} \cdot \mathrm{d}^{-1}$ y flujo promedio de $5.44 \mathrm{l} \cdot \mathrm{m}^{-}$ ${ }^{2} \mathrm{~h}^{-1}$. Los resultados demostraron que las triazinas y el linurón presentan menor grado de eliminación, siendo los mejores resultados a TRC de 60 días con valores entre 73 y $82 \%$; los organoclorados se reducen en el orden de 90 y $99 \%$. Esta tecnología puede alcanzar una calidad óptima del efluente, 
permitiendo su reutilización, o bien puede contribuir en el mantenimiento del estado ecológico adecuado del medio receptor.

Palabras clave: biorreactor de membrana, demanda química de oxígeno, microcontaminantes, pesticidas, tiempo de retención celular, biomasa heterótrofa.

Recibido: $13 / 08 / 2017$

Aceptado: $16 / 03 / 2018$

\section{Introducción}

Actualmente, en el tratamiento de las aguas residuales urbanas no se contempla la eliminación de algunos compuestos orgánicos en concentraciones bajas, también llamados microcontaminantes (MCs), lo que puede suponer la contaminación de las aguas superficiales y subterráneas por algunos MCs tóxicos (agentes tensioactivos, residuos farmacéuticos, productos de cuidado personal, diversos productos químicos de uso doméstico y pesticidas) (Margot, Rossi, Barry, \& Holliger, 2015). Además, la presencia de estos compuestos en los efluentes de las plantas de tratamiento de aguas residuales (PTAR) limita los posibles usos de los efluentes mediante la reutilización. La aparición de estos MCs en el medio acuático se ha convertido en un problema mundial de creciente preocupación medioambiental (Luo et al.,2014) debido a su introducción diaria al medio ambiente en muy bajas concentraciones, principalmente a través de los efluentes de las PTAR. Las PTAR actuales no están diseñadas para eliminar/degradar los MCs, por lo tanto, muchos de estos MCs son capaces de pasar a través de los procesos de tratamiento de aguas residuales, en virtud de su persistencia y/o la introducción continua al medio (Bolong, Ismail, Salim, \& Matsuura, 2009; Luo et al., 2014).

La presencia y el comportamiento de los pesticidas en las PTAR se ha estudiado poco (Buttiglieri, Migliorisi, \& Malpei, 2011), quizás debido a que estas sustancias han sido consideradas de uso agrícola más que de origen urbano (Köck-Schulmeyer et al., 2013). La presencia de pesticidas en las PTAR urbanas se debe principalmente a usos no agrícolas. La lista de tales usos 
incluye la aplicación en la gestión de la hierba, en el control de la vegetación en las industrias y en cultivos no agrícolas, como la silvicultura comercial y horticultura.

Los efectos negativos que pueden generar dichas sustancias son la toxicidad acuática, alteraciones endocrinas, genotoxicidad y aumento de la resistencia de las bacterias patógenas (Halling-Sørensen et al., 1998; Kümmerer, 2004), generando así una creciente preocupación en la comunidad científica; razones que impulsan a buscar tecnologías físico-químicas y biológicas que mineralicen o degraden estas sustancias presentes en las aguas residuales.

Los biorreactores de membrana (MBR, por sus siglas en inglés) combinan el tratamiento biológico (generalmente aeróbico) con la filtración por membrana, para producir efluentes clarificados y en gran parte desinfectados (Judd, 2016). Los MBR se han convertido en una tecnología de última generación que puede emplearse como una alternativa en los procesos de tratamiento de aguas residuales convencionales (Aslam, Charfi, Lesage, Heran, \& Kim, 2017), donde se requiere una alta calidad del agua tratada, en particular para su reutilización, y donde el espacio es limitado (Aslam et al., 2017; Judd, 2016). Los MBR pueden ser capaces de eliminar de forma eficaz un amplio espectro de MCs, que incluye compuestos resistentes al proceso de lodos activados (Luo et al., 2014), además de su alta capacidad y eficiencia de biodegradación, así como baja producción de lodos, bajo costo y simplicidad de construcción (Karaolia et al., 2017).

A lo largo de la última década ha aumentado el interés en la capacidad de los MBR para eliminar MCs. La gran mayoría de investigaciones realizadas con MBR se basa en la eliminación/degradación del grupo de los fármacos y productos de cuidado personal (Bo, Urase, \& Wang, 2009; Kantiani et al., 2008; Kim, Cho, Kim, Vanderford, \& Snyder, 2007; Kimura, Hara, \& Watanabe, 2005); no obstante, algunas investigaciones hacen referencia a los pesticidas de manera general (Ghoshdastidar \& Tong, 2013; Navaratna et al., 2012; Trinh et al., 2016). El MBR se opera con un largo tiempo de retención celular (TRC), que puede mejorar la eliminación de algunos MCs por adsorción en los lodos y su posterior biodegradación. Un TRC prolongado también puede favorecer la proliferación de bacterias de crecimiento lento, mejorando la diversidad microbiana en el reactor, así como una mejor biodegradación de los MCs (Navaratna et al., 2012; Radjenović, Petrović, \& Barceló, 2009; Reif, Suárez, Omil, \& Lema, 2008). Los mecanismos de eliminación de MCs mediante MBR son complejos e incluyen biotransformación, adsorción en la biomasa, volatilización, adsorción en la membrana y retención física por la membrana (Trinh et al., 2016). 
En consecuencia, este documento presenta un estudio sobre la influencia del tiempo de retención celular en la eficiencia de un MBR a escala de laboratorio, en condiciones normales de funcionamiento, y cuyo objetivo es evaluar la eliminación de pesticidas, el rendimiento heterótrofo de la biomasa y la oxidación de la materia orgánica.

\section{Materiales y métodos}

El sistema MBR a escala de laboratorio (iError! No se encuentra el origen de la referencia.) consiste en un reactor de 90 litros (88L útiles), con un módulo de membranas sumergido interno, fibra hueca de microfiltración, Porous Fibers Micronet $R$, de fluoruro de polivinilideno (PVDF), con un tamaño de poro de $0.4 \mu \mathrm{m}$ y una superficie filtrante de $1 \mathrm{~m}^{2}$, un depósito de alimentación y uno de permeado. La alimentación y extracción del permeado a través de las membranas se realizó con bombas peristálticas Dosiper C1R y Watson-Marlow modelo 323 U/D, respectivamente.

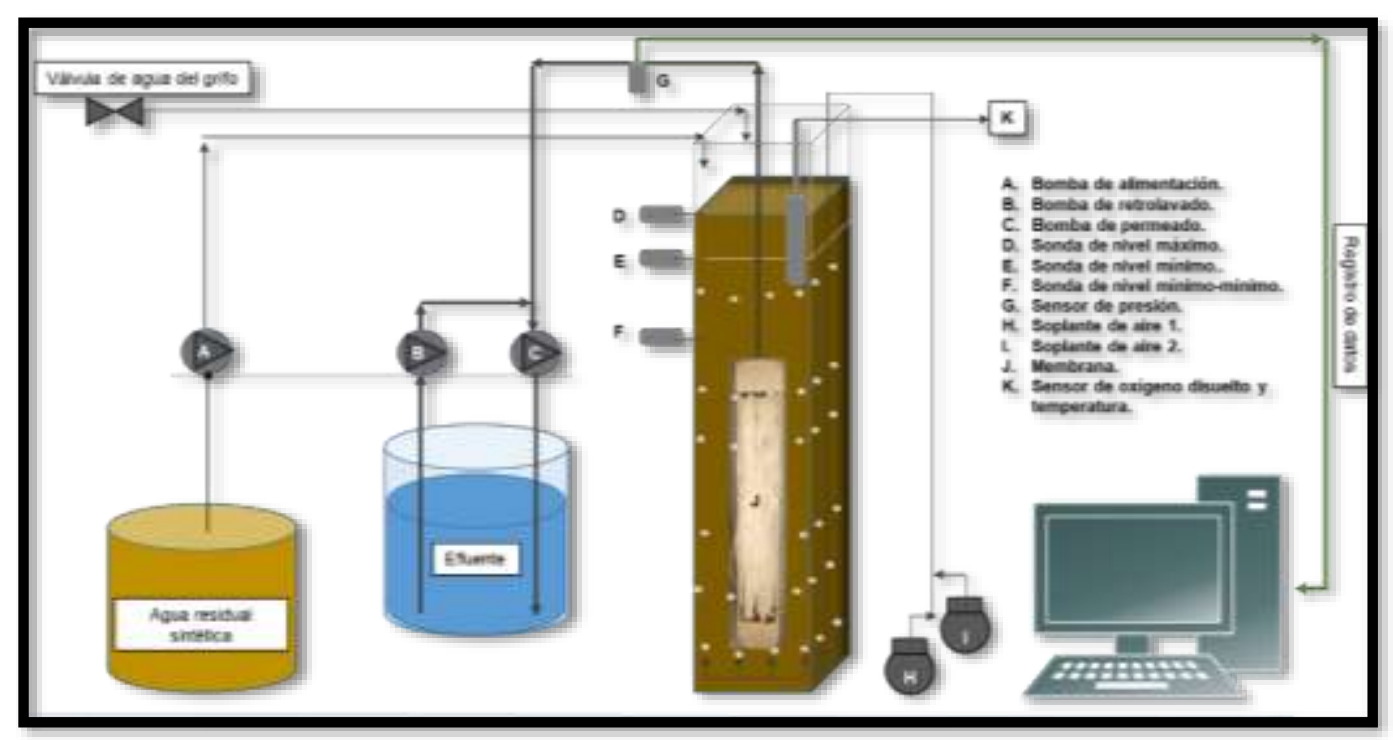

Figura 1. Esquema de la planta piloto. 
Las principales variables de operación fueron monitorizadas en línea y gráficamente en un equipo de cómputo acoplado al sistema. El sistema informático actúa sobre los sensores de nivel de líquido (Endress+Hauser, modelo Liquiphant T FTL260), el transmisor de presión (Cerabar T PMC131), las bombas, las soplantes de aireación (Aqua Medic modelo Mistral 4000) y la sonda (Endress+Hauser, modelo Oxymax COS61) de oxígeno disuelto (OD) y temperatura para mantener las diferentes operaciones de la planta automatizadas.

La filtración va desde el exterior hasta el interior de las membranas (sentido out-in), en un periodo de 10 minutos. Parte de este permeado se almacena en un depósito para ser utilizado en los retrolavados que se le realizan a las membranas durante 30 segundos, como limpieza física.

Las soplantes de aireación estuvieron operando de forma permanente, manteniendo, mediante el burbujeo, la limpieza tangencial de las membranas. De manera paralela, a través de la oxigenación, se le suministró a la biomasa la concentración del oxígeno disuelto (OD) que necesitaba para su crecimiento y se mantenía el licor mezcla en suspensión.

La planta operaba a TRC de 30 y 60 días, a temperatura ambiente (entre 20 y $29^{\circ} \mathrm{C}$ ), con concentraciones de oxígeno de $5 \pm 1 \mathrm{mg} \cdot \mathrm{l}^{-1}$, carga másica promedio de $0.23 \mathrm{kgDQO} / \mathrm{kgSSV}^{-1} \cdot \mathrm{d}^{-1}, \mathrm{pH}$ entre 6.8 y 7.5 , tiempo de retención hidráulico $(\mathrm{TRH})$ de $20 \mathrm{~h}$, flujo promedio de $5.44 \mathrm{~L} \cdot \mathrm{m}^{-2} \mathrm{~h}^{-1} \mathrm{y}$ un incremento de presión transmembrana de $0.83 \mathrm{kPa} \cdot \mathrm{d}^{-1}, 0.38 \mathrm{kPa} \cdot \mathrm{d}^{-1}$ y $0.37 \mathrm{kPa} \cdot \mathrm{d}^{-1}$ durante el periodo de estabilización, TRC de 30 d y TRC de 60 d, respectivamente.

\section{Calidad del agua a tratar}

El agua de alimentación se simula mediante agua sintética, preparada en forma de concentrado, a partir de la composición del alimento sintético recomendada por DIN 38 412-L24 y utilizada por Holler y Trösch (2001), con una DQO de referencia de $3038 \mathrm{mgO}_{2} \cdot \mathrm{l}^{-1}$, como se detalla en la Tabla 1.

Tabla 1. Composición del agua residual sintética.

\begin{tabular}{|l|c|c|}
\hline \multirow{2}{*}{ Compuesto } & DQO referencia & DQO preparada \\
\cline { 2 - 3 } & $3038 \mathrm{mgO}_{2} \cdot \mathrm{I}^{-1}$ & $13500 \mathrm{mgO}_{2} \cdot \mathrm{I}^{-1}$ \\
\hline
\end{tabular}




\begin{tabular}{|c|c|c|}
\hline & $\mathbf{1 ~ I}$ & 30 I \\
\hline Peptona de caseína/g & 1.6 & 177.75 \\
\hline Extracto de carne/g & 1.1 & 122.20 \\
\hline Urea/g & 0.3 & 33.33 \\
\hline $\mathrm{MgSO}_{4} \cdot 7 \mathrm{H}_{2} \mathrm{O} / \mathrm{g}$ & 0.02 & 2.22 \\
\hline $\mathrm{KH}_{2} \mathrm{PO}_{4} / \mathrm{g}$ & 0.28 & 31.11 \\
\hline $\mathrm{CaCl}_{2} \cdot 2 \mathrm{H}_{2} \mathrm{O} / \mathrm{g}$ & 0.04 & 4.44 \\
\hline $\mathrm{NaCl}_{\mathrm{g}}$ & 0.07 & 7.78 \\
\hline $\mathrm{NaHCO}_{3} / \mathrm{g}$ & 3.038 & 13.50 \\
\hline
\end{tabular}

En este estudio se adicionaron en el agua sintética (influente) los microcontaminantes seleccionados que se muestran en la Tabla 2, pertenecientes a las familias de triazinas y organoclorados.

Tabla 2. Microcontaminantes dopados en el influente.

\begin{tabular}{|c|c|c|c|c|c|}
\hline \multicolumn{6}{|c|}{ Organoclorados } \\
\hline Compuesto & $\begin{array}{l}\text { Número } \\
\text { de registro } \\
\text { CAS }\end{array}$ & $\begin{array}{l}\text { Fórmula } \\
\text { molecular }\end{array}$ & Compuesto & $\begin{array}{l}\text { Número } \\
\text { de } \\
\text { registro } \\
\text { CAS }\end{array}$ & $\begin{array}{l}\text { Fórmula } \\
\text { molecular }\end{array}$ \\
\hline Alacloro & $15972-60-8$ & $\begin{array}{c}\mathrm{C}_{14} \mathrm{H}_{20} \mathrm{CINO} \\
2\end{array}$ & a-endosulfan & $959-98-8$ & $\mathrm{C}_{9} \mathrm{H}_{6} \mathrm{Cl}_{6} \mathrm{O}_{3} \mathrm{~S}$ \\
\hline Lindano & $58-89-9$ & $\mathrm{C}_{6} \mathrm{H}_{6} \mathrm{Cl}_{6}$ & $\beta$-endosulfan & $\begin{array}{c}33213-65- \\
9\end{array}$ & $\mathrm{C}_{9} \mathrm{H}_{6} \mathrm{Cl}_{6} \mathrm{O}_{3} \mathrm{~S}$ \\
\hline Heptacloro & $76-44-8$ & $\mathrm{C}_{10} \mathrm{H}_{5} \mathrm{Cl}_{7}$ & Dieldrín & $60-57-1$ & $\mathrm{C}_{12} \mathrm{H}_{8} \mathrm{Cl}_{6} \mathrm{O}$ \\
\hline $\begin{array}{l}\text { Epóxido de } \\
\text { Heptacloro }\end{array}$ & $1024-57-3$ & $\mathrm{C}_{10} \mathrm{H}_{5} \mathrm{Cl}_{7} \mathrm{O}$ & Endrín & $72-20-8$ & $\mathrm{C}_{12} \mathrm{H}_{8} \mathrm{Cl}_{6} \mathrm{O}$ \\
\hline Isodrina & $465-73-6$ & $\mathrm{C}_{12} \mathrm{H}_{8} \mathrm{Cl}_{6}$ & Trifluralina & $1582-09-8$ & $\mathrm{C}_{13} \mathrm{H}_{16} \mathrm{~F}_{3} \mathrm{~N}_{3} \mathrm{O}_{4}$ \\
\hline$p, p-D D D$ & $72-54-8$ & $\mathrm{C}_{14} \mathrm{H}_{10} \mathrm{Cl}_{4}$ & Linuron & $330-55-2$ & $\mathrm{C}_{0} \mathrm{H}_{1} \mathrm{Cl}_{2} \mathrm{~N}_{2} \mathrm{O}_{2}$ \\
\hline $0, p-D D D$ & $53-19-0$ & $\mathrm{C}_{14} \mathrm{H}_{10} \mathrm{Cl}_{4}$ & Lा1िया & 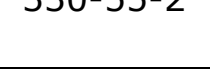 & C9ח110Сl2IV2U2 \\
\hline \multicolumn{6}{|c|}{ Triazinas } \\
\hline Simazina & $122-34-9$ & $\mathrm{C}_{7} \mathrm{H}_{12} \mathrm{~N}_{5} \mathrm{Cl}$ & \multirow{2}{*}{ Terbutilazina } & \multirow{2}{*}{$5915-41-3$} & \multirow{2}{*}{$\mathrm{C}_{9} \mathrm{H}_{16} \mathrm{ClN}_{5}$} \\
\hline Atrazina & $1912-24-9$ & $\mathrm{C}_{8} \mathrm{H}_{14} \mathrm{ClN}_{5}$ & & & \\
\hline
\end{tabular}


Para fijar la concentración inicial de estos microcontaminantes se efectuaron ensayos de toxicidad con el método utilizado por Prieto-Rodríguez et al. (2013) al lodo inóculo procedente de la Estación Depuradora de Rincón de León (Alicante, España). De los resultados obtenidos se concluyó que una concentración de $10 \mu \mathrm{g} \cdot \mathrm{I}^{-1}$ no resultó inhibitoria en la actividad microbiana, siendo además similar a la encontrada en las aguas residuales urbanas (Robles-Molina, Gilbert-López, García-Reyes, \& Molina-Díaz, 2014), por lo que se seleccionó esta concentración inicial de $10 \mu \mathrm{g} \cdot \mathrm{l}^{-1}$ de cada compuesto. Para adicionar los pesticidas al agua residual sintética, se realizó un periodo de estabilización del sistema MBR de 41 días.

\section{Métodos analíticos}

- La DQO se cuantificó por determinación fotométrica de la concentración del cromo (III), tras oxidación de dos horas con $\mathrm{K}_{2} \mathrm{Cr}_{2} \mathrm{O}_{7} / \mathrm{H}_{2} \mathrm{SO}_{4} / \mathrm{Ag}_{2} \mathrm{SO}_{4}$ a $148{ }^{\circ} \mathrm{C}$, utilizando Test de viales de digestión Macherey-Nagel y espectrofotómetro Nanocolor 500D.

- Las pruebas respirométricas se llevaron a cabo utilizando un respirómetro de tipo batch (Spanjers, Vanrolleghem, Olsson, \& Dold, 1996). El respirómetro utilizado es el Modelo BM-T Surcis SL. Este analizador puede medir la tasa dinámica de absorción de oxígeno, $\mathrm{RS}\left(\mathrm{mgO}_{2} \cdot \mathrm{l}^{-1} \mathrm{~h}^{-1}\right)$, la tasa de absorción de oxígeno, OUR $\left(\mathrm{mgO}_{2} \cdot \mathrm{l}^{-1} \mathrm{~h}^{-1}\right)$, consumo de oxígeno, OC $\left(\mathrm{mg} \mathrm{O}_{2} \cdot \mathrm{l}^{-1}\right)$ y otros parámetros.

Los ensayos respirométricos se realizaron tomando una muestra de un litro del licor mezcla, MBR, aplicándosele aireación, utilizando una bomba de aire y un difusor poroso durante $24 \mathrm{~h}$, para alcanzar las condiciones endógenas antes de comenzar el experimento; 30 minutos antes de iniciar el ensayo se le adicionó un inhibidor de la nitrificación (Allil Tiourea, $3 \mathrm{mg} \cdot \mathrm{gSSVLM}^{-1}$ ).

El coeficiente de descomposición de la biomasa $\left(K_{d}\right)$ se evaluó a través de la tasa de absorción de oxígeno endógeno y la concentración total de sólidos en suspensión volátiles en el licor mezcla (SSVLM), según lo descrito por Ramalho (1990) en la ecuación (1).

$K_{d}=\frac{\text { OUR }_{\text {end }}}{1.42[S S V L]}$ 
Para determinar el coeficiente de rendimiento de la biomasa heterótrofa $\left(Y_{H}\right)$ se preparó una disolución madre de acetato de sodio $\left(500 \mathrm{mg} \cdot \mathrm{I}^{-1}\right)$. A partir de ésta se preparan como mínimo tres diluciones más de DQO 100, 200 y 400 $\mathrm{mg} \cdot \mathrm{I}^{-1}$, y mediante test de viales se obtuvo el valor de la DQO total de las diluciones $\left(\mathrm{DQO}_{\mathrm{ac}}\right)$. Posteriormente, se realizó un ensayo Rs al licor mezcla con $50 \mathrm{ml}$ de cada dilución, con el fin de obtener el oxigeno consumido (OC). Así, se puede estimar $Y_{H}$ a partir de la concentración de sustrato y el OC. Fue necesario considerar el factor de conversión $f_{c v}$, con un valor de $1.48 \mathrm{mgDQO} \cdot \mathrm{mgSSVLM}^{-1}$, para evaluar $Y_{H}$, realizando los cálculos según Leyva-Díaz et al. (2013): $Y_{H, D Q O}=1-\frac{O C_{\text {acetato }}}{D Q O_{\text {acetato }}}$ (coeficiente de rendimiento de la biomasa heterótrofa relativo a la demanda química de oxígeno) y $Y_{H}=\frac{Y_{H, D Q O}}{1.48}$ (coeficiente de rendimiento de la biomasa heterótrofa relativo a la concentración de microorganismos).

La adquisición y visualización de los datos se realizó utilizando el software BMAdvance, que generó los respirogramas correspondientes a los diferentes experimentos.

- Para determinar las concentraciones de los MCs se analizaron los siguientes compuestos: alacloro, lindano, heptacloro, epóxido de heptacloro, a-endosulfán, $\beta$-endosulfán, dieldrina, endrina, isodrina, ppDDD, op-DDD, trifluralina, linurón (organoclorados) y simazina, atrazina y terbutilazina (triazinas). Para la cuantificación y análisis de estos microcontaminantes se tomaron muestras del efluente MBR en botellas esterilizadas de $500 \mathrm{ml}$. El acondicionamiento de la muestra consistió en una filtración con filtros Millipore, de $0.7 \mu \mathrm{m}$ de fibra de vidrio; se llevó a cabo la extracción en fase sólida con el equipo Dionex Auto Trace 280 (Thermo Scientific), empleando cartuchos Oasis HLB $6 \mathrm{cc} / 200 \mathrm{mg}$ y disolventes calidad HPLC (diclorometano, acetonitrilo y agua de Sigma Aldrich) según De-Almeida-Azevedo, Lacorte, Vinhas, Viana y Barceló (2000). Para favorecer la retención de los compuestos con menor coeficiente log kow, se llevaron a pH 4 las muestras con ácido clorhídrico/sulfúrico. El extracto recogido en cada tubo se secó con flujo de $N_{2}$ y una vez reducido el volumen se trasvasó la muestra a un "insert" de $200 \mu \mathrm{l}$, donde se continuó el proceso hasta secado total, luego se reconstituyó la muestra con $200 \mu \mathrm{l}$ de disolución de patrón interno (500 $\mu \mathrm{g} \cdot \mathrm{l}^{-1}$ en trifenilfosfato y atrazina-d5). Las muestras se analizaron mediante cromatografía de gases acoplada a espectrometría de masas. El equipo utilizado fue el cromatógrafo modelo Agilent $7890 \mathrm{~A}$ y espectrómetro de masas tipo cuadrupolo modelo Agilent 5975C. La columna utilizada fue la Agilent 19091S-433 HP-5MS (5\% diphenyl-95\% 
dimethylpolysiloxane); columna capilar (30 m $\times 0.25 \mathrm{~mm} \mathrm{DI}, d f=0.25$ $\mu \mathrm{m})$. Se empleó una fase móvil de helio $\left(1.3 \mathrm{ml} \cdot \mathrm{min}^{-1}\right)$ y se trabajó en modo SIM (cuantificación con ion principal e identificación con iones de confirmación).

\section{Resultados y discusión}

\section{Actividad de la biomasa}

Las pruebas respirométricas permitieron medir la actividad de la biomasa heterótrofa durante el periodo experimental. En la Tabla 3 se presentan los valores medios de los parámetros biocinéticos evaluados a partir de la información obtenida en los respirogramas.

Tabla 3. Valores medios de los parámetros biocinéticos.

\begin{tabular}{|c|c|c|c|c|}
\hline \multicolumn{5}{|c|}{ Condiciones endógenas } \\
\hline Parámetro & $\begin{array}{c}\text { OUR } \\
\left(\mathrm{mg} \mathrm{O}_{2} \cdot \mathrm{l}^{-1} \mathrm{~h}^{-}\right. \\
1)\end{array}$ & $\begin{array}{c}\text { SOUR } \\
\left(\mathrm{mgO}_{2} \cdot \mathrm{g} \mathrm{SSVLM}\right. \\
\left.\mathbf{1}^{-1}\right)\end{array}$ & $\begin{array}{c}\mathbf{K d} \\
\left(\mathbf{d}^{-1}\right)\end{array}$ & $\begin{array}{c}Y_{H} \\
(m g \\
\text { SSVLM-mg } \\
\left.\text { DQO-1) }^{-1}\right)\end{array}$ \\
\hline$T R C=30 \mathrm{~d}$ & 2.932 & 1.215 & 0.021 & 0.373 \\
\hline$T C R=60 \mathrm{~d}$ & 2.982 & 0.866 & 0.015 & 0.339 \\
\hline
\end{tabular}

La actividad biológica de los lodos se ha estudiado en condiciones endógenas por los parámetros de absorción de oxígeno (OUR) y la tasa específica de absorción de oxígeno (SOUR). El OUR está directamente asociado con la eliminación del sustrato; sin embargo, el parámetro SOUR está asociado con la eliminación de sustrato y además, con la producción de biomasa, por lo que es un mejor indicador de la actividad biológica del lodo. 
Los sólidos en suspensión en el licor mezcla (SSLM) mostraron una concentración promedio de 3.07 y $2.13 \mathrm{~g} \cdot \mathrm{l}^{-1}$ para TRC de 60 y $30 \mathrm{~d}$, respectivamente. Esto se puede explicar debido a que en esta investigación se ha operado a una carga másica $(\mathrm{Cm})$ baja de $0.23 \mathrm{kgDQO} \cdot \mathrm{KgSSV}^{-1} \cdot \mathrm{d}^{-1}$ y podría corroborarse con los valores bajos de producción de biomasa obtenidos $\left(\mathrm{Y}_{\mathrm{H}}=\right.$ 0.34-0.37), como se demuestra en la Tabla 3. En este sentido, Leyva-Díaz (2015) reportó un valor de $Y_{H}\left(\mathrm{mg} \mathrm{SSVLM} \cdot \mathrm{mg} \mathrm{DQO}^{-1}\right)=0.5040$ en MBR, valores que por lo común se suelen determinar en MBR. En las $\mathrm{Cm}$ bajas, por lo general el crecimiento de microorganismos es limitado por el suministro de nutrientes, lo que implica una menor producción de lodos. En la literatura se encuentra que con el incremento de TRC, las características de bioactividad del lodo en el MBR disminuyen (Ouyang \& Liu, 2009).

Los resultados mostraron una relación proporcional entre el OUR y el SOUR para los dos tiempos de retención celular (TRC) estudiados. Esto se debe a que se trabajó en concentraciones muy similares de SSLM. El SOUR de TRC= 30 días fue ligeramente mayor que en TRC $=60$ días; es decir, el SOUR disminuye con el aumento de TRC. Estos resultados confirman que en TRC cortos la biomasa es más activa. Las altas edades del lodo implican requisitos de energía más bajos, ya que el lodo es cada vez más endógeno y la energía se utiliza para el mantenimiento celular y no para el crecimiento bacteriano. La constante de descomposición endógena $\left(k_{d}\right)$ se mantuvo similar para los dos TRC estudiados $\left(k_{d}=0.021-0.015 \mathrm{~d}^{-1}\right)$.

\section{Reducción de los microcontaminantes}

La concentración de cada compuesto en el influente del MBR se mantuvo en una concentración de $10 \mu \mathrm{g} \cdot \mathrm{l}^{-1}$ durante todo el periodo de investigación (representativa de aguas residuales urbanas reales). En la Tabla 4 se muestran las concentraciones medias encontradas en el efluente MBR para los compuestos estudiados a 30 y 60 días de tiempo de retención celular.

Tabla 4. Concentración media de los pesticidas en el efluente.

\begin{tabular}{|c|c|c|c|}
\hline Compuesto & Log Kow & TRC $=30$ días & TRC = 60 días \\
\hline
\end{tabular}


Ciencias $\overrightarrow{\mathrm{v}} \mathrm{Agua}$

\begin{tabular}{|c|c|c|c|c|c|}
\hline & & $\mathbf{N}$ & $\begin{array}{l}\text { Concentración } \\
\text { media }\left(\mu \mathrm{g} \cdot \mathrm{I}^{-1}\right)\end{array}$ & $\mathbf{N}$ & $\begin{array}{c}\text { Concentración } \\
\text { media }\left(\mu \mathrm{g} \cdot \mathrm{I}^{-1}\right)\end{array}$ \\
\hline Trifluralina & $5.34(a)$ & 7 & $<0.025$ & 17 & $<0.025$ \\
\hline Simazina & $2.18^{(a)}$ & 7 & 4.275 & 17 & 2.690 \\
\hline Atrazina & $2.61^{(a)}$ & 7 & 4.726 & 17 & 2.240 \\
\hline Lindano & 3.72 (a) & 7 & 0.845 & 17 & 0.035 \\
\hline Terbutilazina & $3.21^{(a)}$ & 7 & 3.667 & 17 & 1.767 \\
\hline Heptacloro & $6.10^{(a)}$ & 7 & $<0.025$ & 17 & $<0.025$ \\
\hline Alacloro & 3.52 (a) & 7 & 0.978 & 17 & 0.161 \\
\hline Linuron & $3.20^{(a)}$ & 7 & 4.833 & 17 & 1.795 \\
\hline Isodrina & $6.75^{(a)}$ & 7 & $<0.025$ & 17 & $<0.025$ \\
\hline $\begin{array}{l}\text { Epóxido de } \\
\text { Heptacloro }\end{array}$ & $4.98^{(a)}$ & 7 & $<0.025$ & 17 & $<0.025$ \\
\hline a-endosulfan & $3.83^{(a)}$ & 7 & 0.061 & 17 & $<0.025$ \\
\hline Dieldrín & 5.40 (a) & 7 & $<0.025$ & 17 & $<0.025$ \\
\hline$p, p-D D D$ & 6.02 (a) & 7 & $<0.050$ & 17 & $<0.050$ \\
\hline Endrín & 5.20 (a) & 7 & $<0.025$ & 17 & $<0.025$ \\
\hline$\beta$-endosulfan & $3.83^{(a)}$ & 7 & 0.036 & 17 & $<0.025$ \\
\hline $0, p-D D D$ & $5.87^{(a)}$ & 7 & 0.090 & 17 & $<0.050$ \\
\hline
\end{tabular}

(a) http://www.chemspider.com/ (Royal Society of Chemistry, 2018); $N$ = número de muestras analizadas.

Según los resultados obtenidos en este estudio, se alcanzaron distintas eficiencias de eliminación en el MBR, dependiendo de las propiedades de cada compuesto. Algunos compuestos apenas fueron eliminados, como simazina, atrazina, terbutilazina y linurón; otros moderadamente eliminados, como lindano y alacloro; y otros, altamente eliminados, como trifluralina, heptacloro, isodrina, epóxido de heptacloro, a-endosulfan, dieldrín, endrín, $\beta$-endosulfan, $o, p-D D D$ y $p, p-D D D$.

El grado de eliminación de los microcontaminantes estudiados está relacionado, entre otros factores, con los coeficientes de hidrofobicidad (definidos como log $K_{o w}$ ) de todos los compuestos estudiados, como se muestran en la Tabla 4, obtenidos de la Royal Society of Chemistry (2018).

Según Jones, Voulvoulis y Lester (2005), si un producto químico es más hidrofóbico, mayor es la cantidad que se acumula en la fase sólida (por ejemplo, biosólidos); si es más hidrófilo, mayor será la cantidad que 
permanecerá en la fase acuosa. Según el potencial de sorción, se pueden distinguir tres rangos:

- Log $K_{\text {ow }}<2.5$ bajo potencial de sorción.

- $\log K_{\text {ow }}>2.5$, pero $<4.0$ potencial de sorción media.

- Log $K_{\text {ow }}>4.0$ alto potencial de sorción.

En la iError! No se encuentra el origen de la referencia. se ha representado la eficiencia de eliminación obtenida para los compuestos a TRC de 30 y 60 días en el MBR y está relacionada con la hidrofobicidad de los compuestos ( $\left.\log K_{\text {ow }}\right)$.

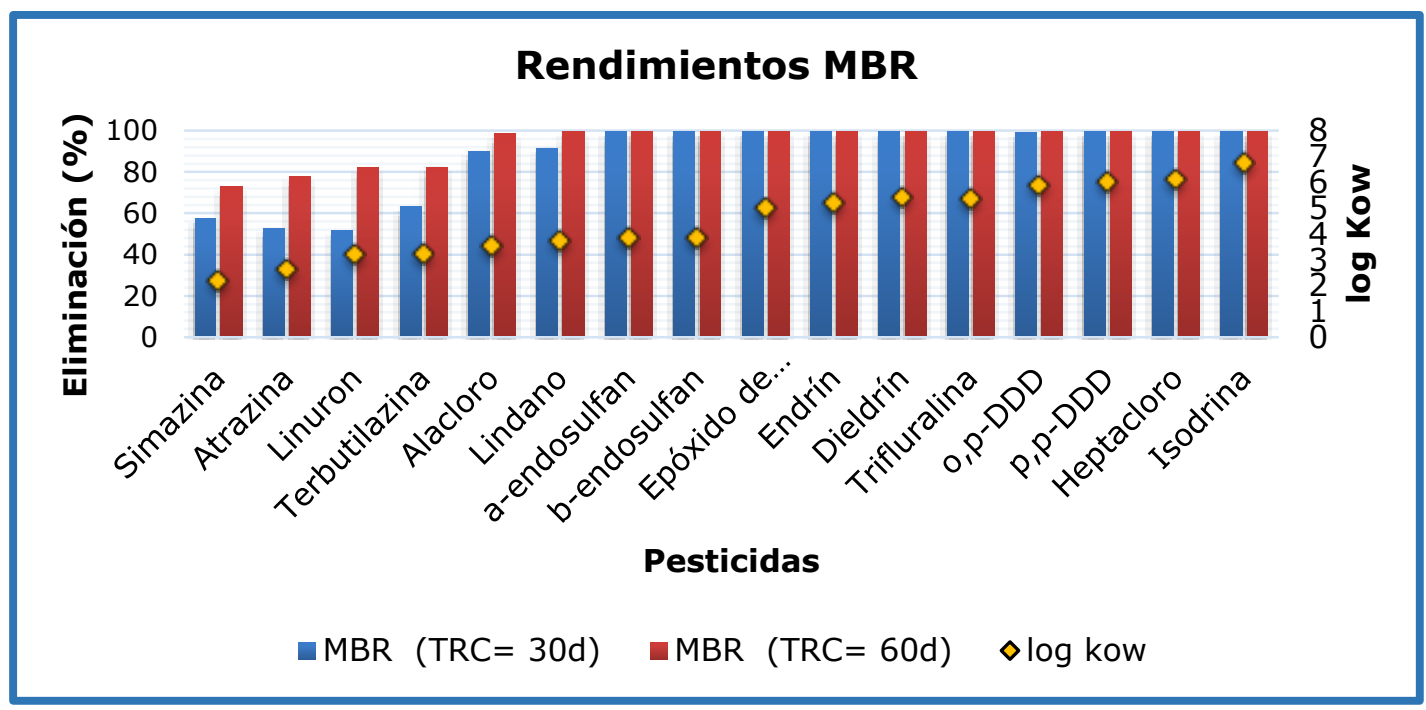

Figura 2. Eliminación media de los pesticidas.

Para los compuestos atrazina, simazina, terbutilazina y linurón a TRC de 30 días, los porcentajes medios de eliminación fueron 52.7, 57.2, 63.3 y 51.7\%, respectivamente; mientras que a TRC de 60 días, los porcentajes medios de eliminación aumentaron con respecto a TRC de 30 días, siendo para estos compuestos $77.6,76.1,82.3$ y $82.1 \%$, respectivamente. La iError! No se encuentra el origen de la referencia. también muestra que estos compuestos presentan coeficientes de hidrofobicidad bajos, lo que indica su marcada característica hidrofílica. Además, en su estructura molecular, tales 
compuestos presentan grupos funcionales aceptores de electrones (cloruros y el anillo s-triazinas para las triazinas), que por su naturaleza tienen una baja biodegradabilidad (Tadkaew, Hai, McDonald, Khan, \& Nghiem, 2011). Todas estas características les permiten ser refractarios al tratamiento MBR, permaneciendo en la fase acuosa. Bernhard, Müller y Knepper (2006) corroboraron que la simazina y atrazina son poco eliminadas en sistemas biológicos debido a su baja hidrofobicidad y su limitada biodegradabilidad. Sin embargo, con el aumento del tiempo de retención celular se puede resaltar un aumento en la eficiencia de eliminación de dichos pesticidas.

En la literatura, otros autores informan un menor rendimiento en la operación MBR, como Wijekoon et al. (2013), con 36\% de reducción de atrazina usando membranas cerámicas; Tadkaew et al. (2011) obtuvieron en su estudio una reducción de $21 \%$ en la eliminación de linurón utilizando módulos de membrana de ultrafiltración de fibra hueca.

Las eficiencias medias de eliminación del alacloro fueron 90.2 y $98.4 \%$ a 30 y 60 días de retención celular, respectivamente. Las eficiencias medias de eliminación del lindano fueron 91.6 y $99.7 \%$ a 30 y 60 días de retención celular, respectivamente. El alacloro y el lindano, a pesar que en su estructura molecular presentan grupos funcionales ganadores de electrones (cloruros), también tienen un moderado carácter hidrófobo (log $K_{\text {ow }}$ de 3.52 y 3.72 ); la adsorción en el fango puede ser su principal mecanismo de eliminación.

En general, los compuestos más hidrófobos, como a-endosulfan, $\beta$-endosulfan, epóxido de heptacloro, endrín, dieldrín, trifluralina, o,p-DDD, p,p-DDD, heptacloro e isodrina alcanzaron eficiencias medias de eliminación superiores a $99 \%$ en ambos tiempos de retención celular. La absorción en los biosólidos y la biodegradación contribuyeron a su eliminación debido a sus altos coeficientes de hidrofobicidad.

El tiempo de retención celular de 60 días puede mejorar la eficiencia de eliminación de la fase acuosa de los compuestos estudiados con respecto al tiempo de retención celular de 30 días, debido a que un TRC prolongado puede favorecer la proliferación de bacterias de crecimiento lento, mejorando así la diversidad microbiana en el reactor y logrando una mejor biodegradación de Ios MCs. La adsorción y biodegradación pueden ser los principales mecanismos de la eliminación de los microcontaminantes por tratamiento con MBR. Boonyaroj, Chiemchaisri, Chiemchaisri, Theepharaksapan y Yamamoto, (2012) y De-Gusseme, Vanhaecke, Verstraete y Boon (2011) en sus investigaciones también detallan que la eliminación de microcontaminantes orgánicos ha mejorado en virtud de unas condiciones de edad más largas de los lodos. No obstante, la presencia de algunos compuestos en el efluente es 
un indicador de la necesidad de agregar un tratamiento terciario para completar la eliminación/mineralización de los pesticidas.

\section{Reducción de materia orgánica}

Esta investigación se realizó a carga másica promedio de $0.23 \mathrm{kgDQO} / \mathrm{kgSSV}^{-}$ ${ }^{1} \mathrm{~d}^{-1}$, con una demanda química de oxígeno de $363 \pm 54 \mathrm{mgO}_{2} \cdot \mathrm{l}^{-1}$, obteniendo reducciones de materia orgánica, los cuales se muestra en la iError! No se encuentra el origen de la referencia., en donde quedan recogidos los datos de la evolución de la DQO en los tiempos de retención celular estudiados tanto en el influente como en el efluente.

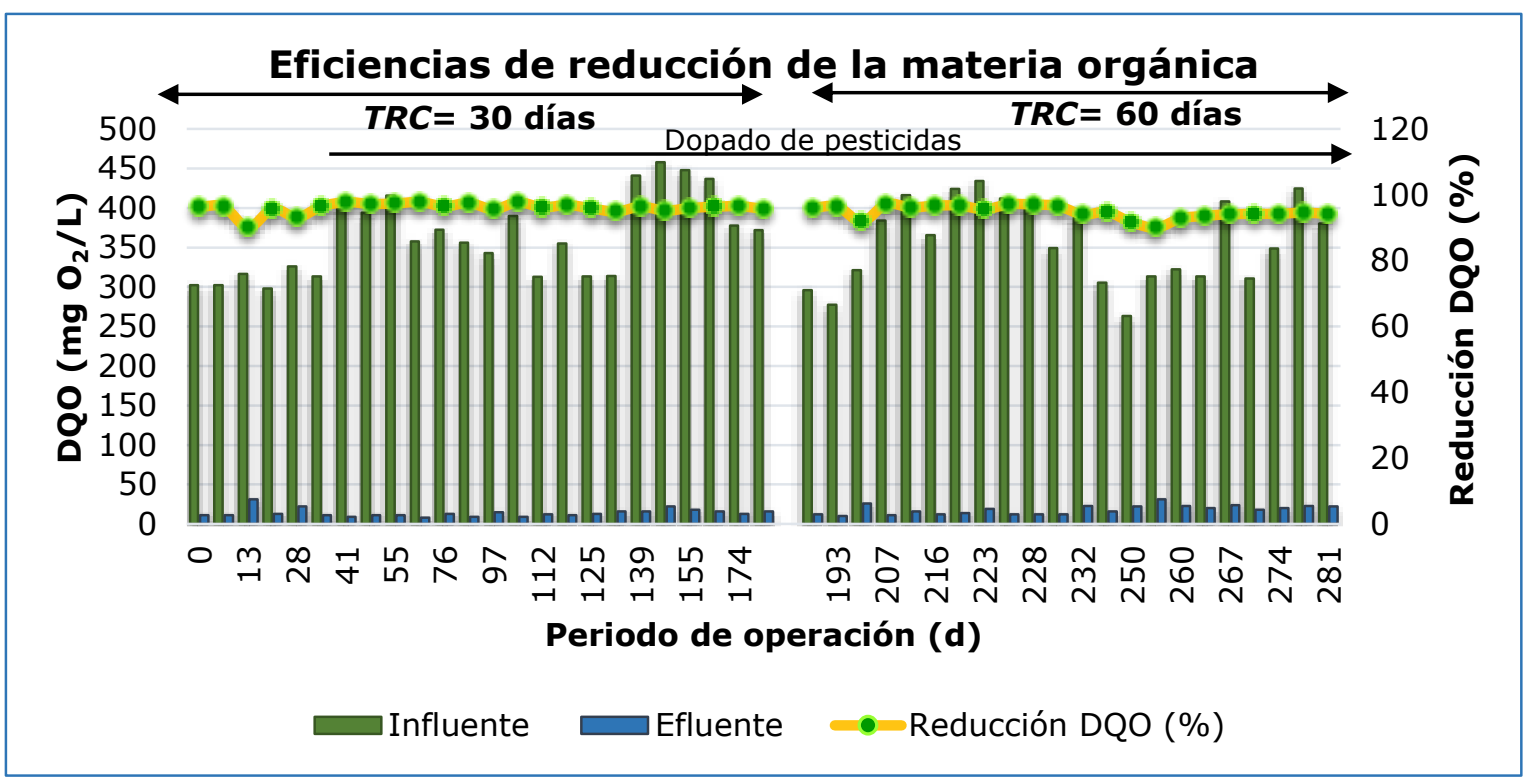

Figura 3. Reducción de la materia orgánica.

Los resultados obtenidos muestran que la eficacia depurativa a lo largo de los dos periodos de estudio fue excelente, obteniéndose un rendimiento medio de eliminación de materia orgánica del $96 \%$. La reducción de la materia orgánica no se ve afectada al dopar los microcontaminantes como se muestra en la iError! No se encuentra el origen de la referencia.. En particular, el día 41 de la investigación se inició el dopado de los pesticidas en el influente, 
obteniendo resultados de reducción de materia orgánica dentro del rango promedio.

Este buen rendimiento de la tecnología MBR se compara con lo encontrado por Neoh, Noor, Mutamim y Lim (2016) en su investigación, que sostiene que el MBR presenta altos rendimientos en la eliminación de materia orgánica y una alternativa atractiva para la reutilización. Domínguez (2010) también realizó estudios de rendimientos de MBR, en donde obtuvo un 97 y $98 \%$ de eliminación de la materia orgánica, y Rodríguez et al. (2011), que obtuvo rendimientos superiores a $90 \%$. Todo esto demuestra la robustez y capacidad de este tipo de tecnología para tratar aguas residuales.

\section{Conclusiones}

Para la familia de los organoclorados se obtuvieron buenos rendimientos de eliminación, con valores entre 90 y $99 \%$ en los dos tiempos de retención celular estudiados.

Para la familia de las triazinas (simazina, atrazina y terbutilazina) y el linurón, la operación a TRC de 60 días supuso un incremento promedio de $20 \%$ de eliminación, alcanzando valores cercanos al $80 \%$ de rendimiento. En la operación de MBR, el incremento del tiempo de retención celular aumentó la eliminación de algunos MCs de la línea de agua.

Mediante el sistema MBR, los compuestos hidrofóbicos pueden alcanzar rendimientos de eliminación superiores a $90 \%$, mientras que los compuestos aceptores de electrones (persistentes) y con hidrofobicidad baja pueden alcanzar eficiencias de eliminación por debajo de $60 \%$.

Los coeficientes de crecimiento heterótrofo presentaron valores bajos debido a que se trabajó a carga baja. Sin embargo, se alcanzaron eficiencias en la reducción de DQO de $96 \%$, lo que demuestra que la presencia de MCs en el influente no afecta a los rendimientos del MBR en la eliminación de materia orgánica.

Estos resultados demuestran la eficiencia de los sistemas MBR en la eliminación/degradación de algunos pesticidas. Para mejorar la eficacia en la eliminación de los microcontaminantes se pueden plantear nuevas 
investigaciones, situando al MBR como una primera barrera, seguida de un postratamiento (procesos de oxidación avanzada y/o procesos de membrana), que mejore la calidad del efluente en cuanto a la presencia de estas sustancias más refractarias.

\section{Agradecimientos}

Esta investigación ha sido desarrollada en el Instituto del Agua y las Ciencias Ambientales de la Universidad de Alicante, en el marco del Proyecto "CTM 2013-46669: eliminación y/o degradación de contaminantes emergentes en aguas mediante tratamientos combinados", del Ministerio de Economía y Competitividad de España, y también con la ayuda del Subprograma de Ayuda Económica Complementaria Nº8-2014-074 del Instituto para la Formación y Aprovechamiento de Recursos Humanos (IFARHU)-Universidad Tecnológica de Panamá, República de Panamá.

\section{Referencias}

Aslam, M., Charfi, A., Lesage, G., Heran, M., \& Kim, J. (2017). Membrane bioreactors for wastewater treatment: A review of mechanical cleaning by scouring agents to control membrane fouling. Chemical Engineering Journal, 307, 897-913. Recuperado de http://dx.doi.org/10.1016/j.cej.2016.08.144

Bernhard, M., Müller, J., \& Knepper, T. P. (2006). Biodegradation of persistent polar pollutants in wastewater: Comparison of an optimised lab-scale membrane bioreactor and activated sludge treatment. Water Research, 40(18), 3419-3428.

Recuperado

de https://doi.org/10.1016/j.watres.2006.07.011

Bo, L., Urase, T., \& Wang, X. (2009). Biodegradation of trace pharmaceutical substances in wastewater by a membrane bioreactor. Frontiers of Environmental Science \& Engineering in China, 3(2), 236-240. Recuperado de https://doi.org/10.1007/s11783-009-0004-9

Bolong, N., Ismail, A. F., Salim, M. R., \& Matsuura, T. (2009). A review of the effects of emerging contaminants in wastewater and options for their removal. Desalination, 239(1-2), 229-246. Recuperado de http://dx.doi.org/10.1016/j.desal.2008.03.020 
Boonyaroj, V., Chiemchaisri, C., Chiemchaisri, W., Theepharaksapan, S., \& Yamamoto, K. (2012). Toxic organic micro-pollutants removal mechanisms in long-term operated membrane bioreactor treating municipal solid waste leachate. Bioresource Technology, 113, 174-180. Recuperado de http://dx.doi.org/10.1016/j.biortech.2011.12.127

Buttiglieri, G., Migliorisi, L., \& Malpei, F. (2011). Adsorption and removal at low atrazine concentration in an MBR pilot plant. Water Science and Technology, 63(7), 1334-1340. DOI: 10.2166 / wst.2011.130

De-Almeida-Azevedo, D., Lacorte, S., Vinhas, T., Viana, P., \& Barceló, D. (2000). Monitoring of priority pesticides and other organic pollutants in river water from portugal by gas chromatography-mass spectrometry and liquid chromatography-atmospheric pressure chemical ionization mass spectrometry. Journal of Chromatography $A, 879(1), 13-26$. Recuperado de https://doi.org/10.1016/S0021-9673(00)00372-1

De-Gusseme, B., Vanhaecke, L., Verstraete, W., \& Boon, N. (2011). Degradation of acetaminophen by delftia tsuruhatensis and pseudomonas aeruginosa in a membrane bioreactor. Water Research, 45(4), 1829-1837. Recuperado de http://dx.doi.org/10.1016/j.watres.2010.11.040

Domínguez, C., L. (2010). Análisis del ensuciamiento de membranas en biorreactores de membranas sumergidas escala piloto (tesis). Universidad de Alicante, Alicante, España.

Ghoshdastidar, A. J., \& Tong, A. Z. (2013). Treatment of 2, 4-D, mecoprop, and dicamba using membrane bioreactor technology. Environmental Science and Pollution Research, 20(8), 5188-5197. Recuperado de https://doi.org/10.1007/s11356-013-1498-z

Halling-Sørensen, B., Nors, N. S., Lanzky, P. F., Ingerslev, F., Holten-Lützhøft, H. C., \& Jørgensen, S. E. (1998). Occurrence, fate and effects of pharmaceutical substances in the environment- A review. Chemosphere, 36(2), 357-393. Recuperado de http://dx.doi.org/10.1016/S00456535(97)00354-8

Holler, S., \& Trösch, W. (2001). Treatment of urban wastewater in a membrane bioreactor at high organic loading rates. Journal of Biotechnology, 92(2), 95-101. Recuperado de https://doi.org/10.1016/S0168-1656(01)00351-0

Jones, O., Voulvoulis, N., \& Lester, J. (2005). Human pharmaceuticals in wastewater treatment processes. Critical Reviews in Environmental 
Science and Technology, 35(4), 401-427. Recuperado de https://doi.org/10.1080/10643380590956966

Judd, S. J. (2016). The status of industrial and municipal effluent treatment with membrane bioreactor technology. Chemical Engineering Journal, 305, 37-45. Recuperado de http://dx.doi.org/10.1016/j.cej.2015.08.141

Kantiani, L., Farré, M., Asperger, D., Rubio, F., González, S., López-de-Alda, M. J., Petrović, M., Shelver, W. L., Barceló, D. (2008). Triclosan and methyl-triclosan monitoring study in the northeast of spain using a magnetic particle enzyme immunoassay and confirmatory analysis by gas chromatography-mass spectrometry. Journal of Hydrology, 361(12), 1-9. Recuperado de https://doi.org/10.1016/j.jhydrol.2008.07.016

Karaolia, P., Michael-Kordatou, I., Hapeshi, E., Alexander, J., Schwartz, T., \& Fatta-Kassinos, D. (2017). Investigation of the potential of a membrane BioReactor followed by solar fenton oxidation to remove antibioticrelated microcontaminants. Chemical Engineering Journal, 310, Part 2, 491-502. Recuperado de http://dx.doi.org/10.1016/j.cej.2016.04.113

Kim, S. D., Cho, J., Kim, I. S., Vanderford, B. J., \& Snyder, S. A. (2007). Occurrence and removal of pharmaceuticals and endocrine disruptors in south korean surface, drinking, and waste waters. Water Research, 41(5), 1013-1021. Recuperado de https://doi.org/10.1016/j.watres.2006.06.034

Kimura, K., Hara, H., \& Watanabe, Y. (2005). Removal of pharmaceutical compounds by submerged membrane bioreactors (MBRs). Desalination, 178(1-3), 135-140. Recuperado de https://doi.org/10.1016/j.desal.2004.11.033

Köck-Schulmeyer, M., Villagrasa, M., López-de-Alda, M., Céspedes-Sánchez, R., Ventura, F., \& Barceló, D. (2013). Occurrence and behavior of pesticides in wastewater treatment plants and their environmental impact. Science of the Total Environment, 458-460, 466-476. Recuperado de http://doi.org/10.1016/j.scitotenv.2013.04.010

Kümmerer, K. (2004). Resistance in the environment. Journal of Antimicrobial Chemotherapy, 54(2), 311-320. Recuperado de https://doi.org/10.1093/jac/dkh325

Leyva-Díaz, J. C. (2015). Estudio cinético de biorreactores de membrana con y sin lecho móvil aplicados al tratamiento de aguas residuales urbanas (tesis). Universidad de Granada, Granada, España. Recuperado de 
https://dialnet.unirioja.es/servlet/tesis?codigo $=57830 \&$ orden $=0 \& i n f o=\mid$ ink

Leyva-Díaz, J. C., Calderón, K., Rodríguez, F. A., González-López, J., Hontoria, E., \& Poyatos, J. M. (2013). Comparative kinetic study between moving bed biofilm reactor-membrane bioreactor and membrane bioreactor systems and their influence on organic matter and nutrients removal. Biochemical Engineering Journal, 77, 28-40. Recuperado de /https://doi.org/10.1016/j.bej.2013.04.023

Luo, Y., Guo, W., Ngo, H. H., Nghiem, L. D., Hai, F. I., Zhang, J., \& Wang, X. C. (2014). A review on the occurrence of micropollutants in the aquatic environment and their fate and removal during wastewater treatment. Science of the Total Environment, 473-474, 619-641. Recuperado de http://dx.doi.org/10.1016/j.scitotenv.2013.12.065

Margot, J., Rossi, L., Barry, D. A., \& Holliger, C. (2015). A review of the fate of micropollutants in wastewater treatment plants. Wiley Interdisciplinary Reviews: Water, 2(5), 457-487. Recuperado de https://doi.org/10.1002/wat2.1090

Navaratna, D., Elliman, J., Cooper, A., Shu, L., Baskaran, K., \& Jegatheesan, V. (2012). Impact of herbicide ametryn on microbial communities in mixed liquor of a membrane bioreactor (MBR). Bioresource Technology, 113, 181-190. Recuperado de https://doi.org/10.1016/j.biortech.2011.12.018

Neoh, C. H., Noor, Z. Z., Mutamim, N. S. A., \& Lim, C. K. (2016). Green technology in wastewater treatment technologies: Integration of membrane bioreactor with various wastewater treatment systems. Chemical Engineering Journal, 283, 582-594. Recuperado de http://dx.doi.org/10.1016/j.cej.2015.07.060

Ouyang, K., \& Liu, J. (2009). Effect of sludge retention time on sludge characteristics and membrane fouling of membrane bioreactor. Journal of Environmental Sciences, 21(10), 1329-1335. Recuperado de https://doi.org/10.1016/S1001-0742(08)62422-5

Prieto-Rodríguez, L., Oller, I., Klamerth, N., Agüera, A., Rodríguez, E., \& Malato, S. (2013). Application of solar AOPs and ozonation for elimination of micropollutants in municipal wastewater treatment plant effluents. Water Research, 47(4), 1521-1528. Recuperado de https://doi.org/10.1016/j.watres.2012.11.002

Radjenović, J., Petrović, M., \& Barceló, D. (2009). Fate and distribution of pharmaceuticals in wastewater and sewage sludge of the conventional 
activated sludge (CAS) and advanced membrane bioreactor (MBR) treatment. Water Research, 43(3), 831-841. Recuperado de https://doi.org/10.1016/j.watres.2008.11.043

Ramalho, R. S. (1990). Tratamiento de aguas residuales. Barcelona, España: Editorial Reverté.

Reif, R., Suárez, S., Omil, F., \& Lema, J. M. (2008). Fate of pharmaceuticals and cosmetic ingredients during the operation of a MBR treating sewage. Desalination, 221(1-3), 511-517. Recuperado de https://doi.org/10.1016/j.desal.2007.01.111

Robles-Molina, J., Gilbert-López, B., García-Reyes, J. F., \& Molina-Díaz, A. (2014). Monitoring of selected priority and emerging contaminants in the guadalquivir river and other related surface waters in the province of jaén, south east spain. Science of the Total Environment, 479-480(1), 247-257. Recuperado

https://doi.org/10.1016/j.scitotenv.2014.01.121

Rodríguez, F. A., Poyatos, J. M., Reboleiro-Rivas, P., Osorio, F., GonzálezLópez, J., \& Hontoria, E. (2011). Kinetic study and oxygen transfer efficiency evaluation using respirometric methods in a submerged membrane bioreactor using pure oxygen to supply the aerobic conditions. Bioresource Technology, 102(10). Recuperado de https://doi.org/10.1016/j.biortech.2011.02.083

Royal Society of Chemistry. (2018). ChemSpinder. Search and share chemetry. London, UK: Royal Society of Chemistry. Recuperado de http://www.chemspider.com/

Spanjers, H., Vanrolleghem, P., Olsson, G., \& Dold, P. (1996). Respirometry in control of the activated sludge process. Water Science and Technology, 34(3-4), 117-126

Tadkaew, N., Hai, F. I., McDonald, J. A., Khan, S. J., \& Nghiem, L. D. (2011). Removal of trace organics by MBR treatment: The role of molecular properties. Water Research, 45(8), 2439-2451. Recuperado de https://doi.org/10.1016/j.watres.2011.01.023

Trinh, T., Van-den-Akker, B., Coleman, H. M., Stuetz, R. M., Drewes, J. E., LeClech, P., \& Khan, S. J. (2016). Seasonal variations in fate and removal of trace organic chemical contaminants while operating a full-scale membrane bioreactor. Science of the Total Environment, 550, 176-183. Recuperado de https://doi.org/10.1016/j.scitotenv.2015.12.083

Wijekoon, K. C., Hai, F. I., Kang, J., Price, W. E., Guo, W., Ngo, H. H., \& Nghiem, L. D. (2013). The fate of pharmaceuticals, steroid hormones, 
phytoestrogens, UV-filters and pesticides during MBR treatment. Bioresource Technology, 144, 247-254. Recuperado de https://doi.org/10.1016/j.biortech.2013.06.097 